\title{
Logic Matrix Design Method for Pan Boolean Algebra PID Control
}

\author{
CHEN Jin ${ }^{1, a}$, Xu Tao ${ }^{2, b}$ \\ ${ }^{1}$ College of Electronic and Electrical Engineering, Shanghai Second Polytechnic \\ University,Shanghai,201209 \\ ${ }^{2}$ College of Electronic and Electrical Engineering, Shanghai Second Polytechnic \\ University,Shanghai,201209 \\ aemail: chenjin@sspu.edu.cn, bemail: xutao@sspu.edu.cn
}

Keywords: Pan -Boolean algebra PID; Pan-logic truth table; Boolean logic matrix

\begin{abstract}
Based on the analyzing for the Pan-logic graph of Pan -Boolean algebra PID control, it is introduced in this paper that a new method of Pan-logic truth table can be used to depict the relations between input and output variables of Pan-Boolean algebra PID controller. By quantizing and grading the controller variable, a set of Boolean logic variable denoted as a Boolean logic vector. There is but a matrix between the controller input logic vector and output logic vector, and the matrix can be solved by the matrix equation of pan-logic truth table. So a novel design method of Pan-Boolean algebra PID controller logic matrix is suggested, it can be applied to general logic matrix construction of Pan-Boolean algebra control system.
\end{abstract}

\section{Introduction}

Pan Boolean algebra control theory originated from the Pan Boolean algebra in the early 80's [1]. After several decades of development, the Pan Boolean algebra control has theory and application of the systematic research achievements in the single variable control system design and control. Nine point control theory systematically discusses the Pan Boolean algebra controller design, dynamic analysis, static characteristics analysis and system stability analysis and control principle [2]. The reference [3] introduces parameter analysis of spatial Pan Boolean algebra of PID control, the 27 control parameters of Pan Boolean algebra of PID controller in the coordinate system used to describe, analysis indexes of the performance of this controller. However, so far as the references[4] mentioned, because of the logic system analysis tool for little, Pan Boolean algebra PID control is designed to determine the parameters of PID controller based on computer simulation, and the lack of universal design method. Making the in-depth study of the theory on how to Pan Boolean algebra PID control logic system, the reference [4] provide a good research ideas and tools.

This paper discusses the structure of logic matrix design problem of Pan Boolean algebra of PID controller, based on the logic truth table is improved, the pan logic relation matrix description logic variables between the true value table, so as to obtain the design method of logic matrix Pan Boolean algebra of PID controllers, the Pan Boolean algebra PID control problem into traditional discrete mathematics problems.

\section{Pan Boolean algebra PID control and logic vector}

Pan Boolean algebra PID control structure diagram as shown in Fig.1, the set value $(R(T))$ equals system run expectations. The system output value is $C(T)$. The deviation: e $(\mathrm{T})=\mathrm{R}(\mathrm{T})-\left(\mathrm{C}(\mathrm{T})\right.$. The deviation integral can be expressed as $\int e(t) d t$, the deviation differential can be expressed as $\frac{d e(t)}{d t}$, the controller output can be expressed as Y (control action). The G(s) is the object transfer function. 


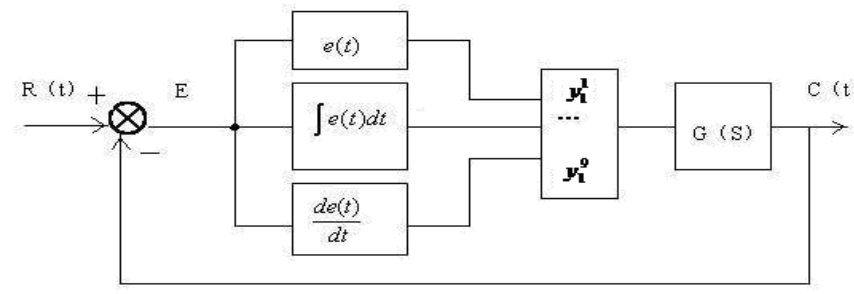

Fig. 1: Pan Boolean algebra PID control principle diagram

Assume that the system deviation, deviation change rate (differential) and the deviation of integral as input, the controller according to certain rules of action, in order to keep the system output in the near set value and stable operation.

Pan Boolean algebra PID control logic diagram shown in Figure 2. The system deviation quantization into three level, let, $x_{1}^{1}, x_{1}^{2}, x_{1}^{3}$ respectively, expressed the deviation is greater than zero (positive, $x_{1}^{1}$ ), in the deviation of zero band (zero, $x_{1}^{2}$ )and less than zero (negative, $x_{1}^{3}$ ) three states, defines a logical vector as $\left.X_{1=[} x_{1}^{1}, x_{1}^{2}, x_{1}^{3}\right]$.

The system deviation differential $\frac{d e}{d t}$ quantization is divided into three levels, let, respectively, $x_{2}^{1}, x_{2}^{2}, \quad x_{2}^{3}$ expressed deviation differential is greater than zero (positive, $x_{2}^{1}$ ), zero (zero, $x_{2}^{2}$ ) and less than zero (negative, $x_{2}^{3}$ ) three states, defines a logical vector $X_{2}=\left[\begin{array}{lll}x_{2}^{1}, & x_{2}^{2}, & x_{2}^{3}\end{array}\right]$, the system deviation integral $\int e d t$ quantization is divided into three levels, let $x_{3}^{1}, x_{3}^{2}, x_{3}^{3}$ respectively, expressed system deviation integral greater than zero (positive, $x_{3}^{1}$ ), zero band (zero ${ }_{3}^{2}$ ) and less than zero (negative, $x_{3}^{3}$ ) three kinds of state, defines a logical vector $X_{3}=\left[x_{3}^{1}, x_{3}^{2}, x_{3}^{3}\right]$; Using symbols, $y_{1}^{1}, y_{1}^{2}, y_{1}^{3}, y_{1}^{4}, y_{1}^{5}, y_{1}^{6}, y_{1}^{7}, y_{1}^{8}, y_{1}^{9}$, describe the system nine kinds of control action rules of state, as follows: add much more $\left(y_{1}^{1}\right)$, add more $\left(y_{1}^{2}\right)$,add a little $\left(y_{1}^{3}\right)$, add a little more $\left(y_{1}^{4}\right)$, remain unchanged $\left(y_{1}^{5}\right)$, decrease much more $\left(y_{1}^{9}\right)$, decrease more $\left(y_{1}^{8}\right)$,decrease a little $\left(y_{1}^{7}\right)$,decrease a little more $\left(y_{1}^{6}\right)$, defines a logical vector $Y_{1=[} y_{1}^{1}, y_{1}^{2}, y_{1}^{3}, y_{1}^{4}$, $\left.y_{1}^{5}, y_{1}^{6}, y_{1}^{7}, y_{1}^{8}, y_{1}^{9}\right]$. By the universal logic in Figure 2 and the Pan Boolean algebra axiom system [1], can get the logic such as equation of Pan Boolean algebra PID controller (1) - (9) shows. However, how to design its Pan algebra PID logical matrix?

$$
\begin{aligned}
& y_{1}^{1}=x_{1}^{1} x_{2}^{2} x_{3}^{3}+x_{1}^{1} x_{2}^{3} x_{3}^{3}+x_{1}^{1} x_{2}^{1} x_{3}^{2} \\
& y_{1}^{2}=x_{1}^{1} x_{2}^{3} x_{3}^{1}+x_{1}^{1} x_{2}^{2} x_{3}^{2}+x_{1}^{1} x_{2}^{1} x_{3}^{3} \\
& y_{1}^{3}=x_{1}^{1} x_{2}^{1} x_{3}^{1}+x_{1}^{1} x_{2}^{2} x_{3}^{1}+x_{1}^{1} x_{2}^{3} x_{3}^{2}+x_{1}^{2} x_{2}^{3} x_{3}^{3} \\
& y_{1}^{4}=x_{1}^{2} x_{2}^{1} x_{3}^{2}+x_{1}^{2} x_{2}^{1} x_{3}^{3}+x_{1}^{2} x_{2}^{2} x_{3}^{3} \\
& y_{1}^{5}=x_{1}^{2} x_{2}^{2} x_{3}^{2} \\
& y_{1}^{6}=x_{1}^{2} x_{2}^{2} x_{3}^{1}+x_{1}^{2} x_{2}^{3} x_{3}^{1}+x_{1}^{2} x_{2}^{3} x_{3}^{2} \\
& y_{1}^{7}=x_{1}^{3} x_{2}^{1} x_{3}^{2}+x_{1}^{3} x_{2}^{2} x_{3}^{3}+x_{1}^{3} x_{2}^{3} x_{3}^{3}+x_{1}^{2} x_{2}^{1} x_{3}^{1} \\
& y_{1}^{8}=x_{1}^{3} x_{2}^{1} x_{3}^{3}+x_{1}^{3} x_{2}^{2} x_{3}^{2}+x_{1}^{3} x_{2}^{3} x_{3}^{1} \\
& y_{1}^{9}=x_{1}^{3} x_{2}^{1} x_{3}^{1} x_{1}^{3} x_{2}^{2} x_{3}^{1}+x_{1}^{3} x_{2}^{3} x_{3}^{2}
\end{aligned}
$$

Obviously, the logic equations (1) - (9) can be represented by matrix into (10), the key is how 
to conceive and design the logic matrix.

$$
\begin{aligned}
& Y_{1}=A X_{1} X_{2} X_{3} \\
& Y_{1}=A_{\ltimes} X_{1 \ltimes} X_{2 \ltimes} X_{3}
\end{aligned}
$$

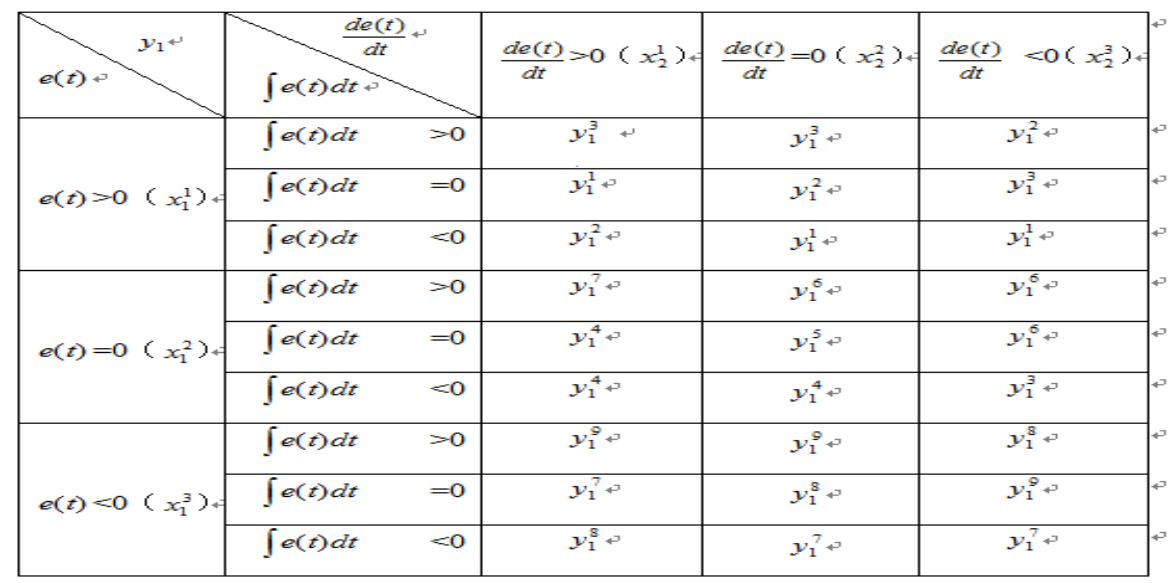

Fig. 2: Pan Pan Boolean algebra PID control logic diagram

\section{Pan Algebra PID controller's Logic Matrix Design}

From the general logic of Figure 2, the relations between input logic vectors $\left(X_{1}=\left[\begin{array}{lll}x_{1}^{1}, & x_{1}^{2}, & x_{1}^{3}\end{array}\right]\right.$, $\left.X_{2}=\left[\begin{array}{lll}x_{2}^{1}, & x_{2}^{2}, & x_{2}^{3}\end{array}\right], \quad X_{3}=\left[\begin{array}{lll}x_{3}^{1}, & x_{3}^{2}, & x_{3}^{3}\end{array}\right]\right)$ is logic and, so this relationship can be expressed in truth value table. The logic relationship between the output logical vector and input logic vector should be expressed with the truth table, so by the pan logic diagram 2 to get its Boolean logic truth table, as shown in table 1.Black solid line in the table 1 represents a logical and relation between the input variables and output variables separated by thin lines. With the traditional algebra of logic truth table comparison, similarities: each variable logic is two valued logic variables, a value of 0 or 1 ; the difference: any time, logic variables in logic vector has only one fetch logic 1 , the rest to take a logic 0 . As with traditional logic truth table phase difference, called the logic truth table for the pan logic truth table. From table 1, the input logic $X_{1}=\left[x_{1}^{1}, x_{1}^{2}, x_{1}^{3}\right] 27$ columns for the logical value with the matrix $A_{1}$ representation , by the same token, matrix $A_{2}$ and $A_{3}$ represents a logical vector $X_{2}=\left[\begin{array}{lll}x_{2}^{1}, & x_{2}^{2}, & x_{2}^{3}\end{array}\right]$ and $X_{3}=\left[\begin{array}{lll}x_{3}^{1}, & x_{3}^{2}, & x_{3}^{3}\end{array}\right] 27$ columns for logical values. The

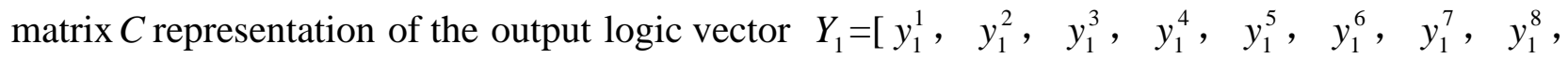
$y_{1}^{9}$ ] 27 columns for logical values. From table 1, the logical relationship between the output logic vector $Y_{1}$ and the input vector $X_{1}, X_{2}, X_{3}$ get the formula (12) are as follows:

$$
C Y_{1}^{T}=A_{1} X_{1}^{T} A_{2} X_{2}{ }^{T} A_{3} X_{3}{ }^{T}
$$

Table1: Pan Boolean algebra PID controller pan logic truth table

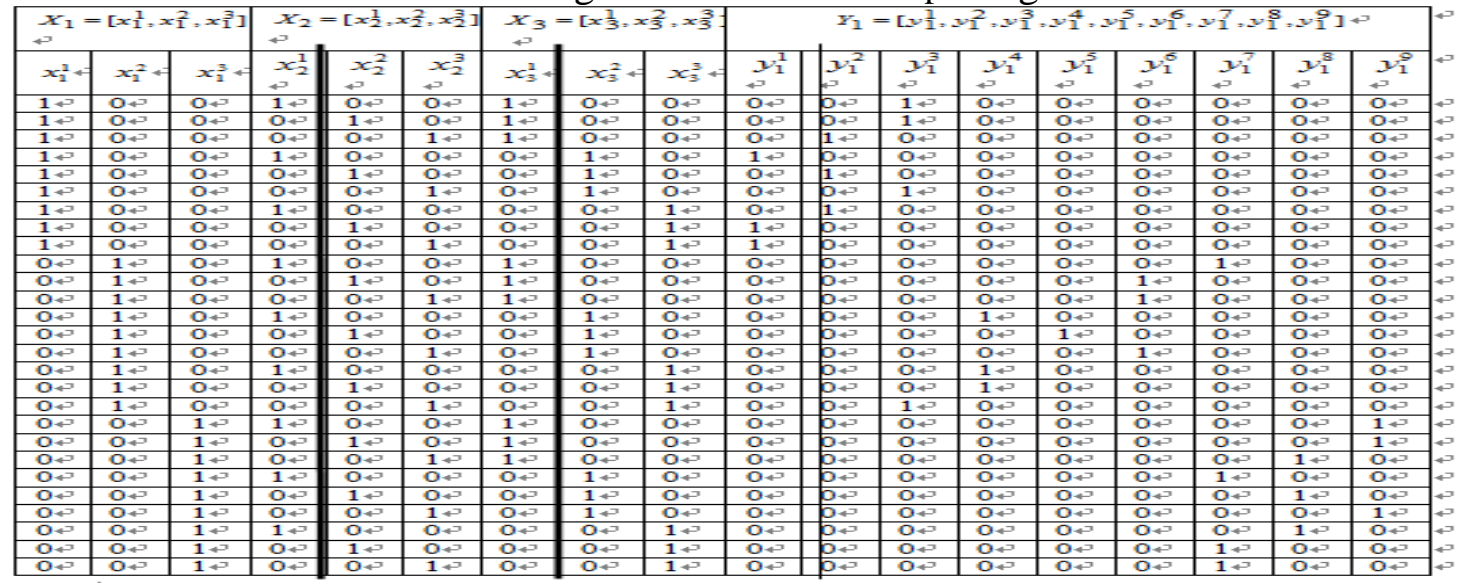


It is not difficult to verify, by formula (12) can get the formula (1) - (9). By the equation (12), by way of the ordinary matrix transform and operation, can get the formula (10) in the matrix $A$.

Now discuss the general situation of Pan Boolean algebra PID controller, Hypothetical bias $e(t)$ quantization into $n 1$ level, expressed as $x_{1}=\left[\begin{array}{lll}x_{1}^{1} & x_{1}^{2}, \ldots, x_{1}^{n 1}\end{array}\right]$, deviation differential $\frac{d e}{d t}$ quantization into $n 2$ level, expressed as $X_{2}=\left[\begin{array}{lll}x_{2}^{1}, & x_{2}^{2}, \ldots, x_{2}^{n 2}\end{array}\right]$, deviation integral $\int e d t$ quantization into n3level, expressed as $X_{3}=\left[\begin{array}{lll}x_{3}^{1}, & x_{3}^{2}, \ldots, x_{3}^{n 3}\end{array}\right]$, the output Y quantization into $n 4$ level, expressed as $Y_{1}=\left[y_{1}^{1}, y_{1}^{2}, \ldots, y_{1}^{n 4}\right]$. Similarly, the formula (12) was established, where $C$ is a matrix of order $n \times n 4$, is $A_{1}$ a matrix of order $n \times n 1, A_{2}$ is a matrix of order $n \times n 2, A_{3}$ is a matrix of order $n \times n 3, n=n 1 \times n 2 \times n 3$.

Constructing the matrix $C, A_{1}, A_{2}, A_{3}$ methods: by means of Pan logic diagram lists of Pan logic truth table, by the universal logic truth table is obtained. When the grading matrix ranks parameter input variable number is not the same $(n 1 \neq n 2 \neq n 3)$, the matrix dimension of equations (12) are different, do not use ordinary matrix multiplication rule to solve the matrix operation, we need to use a semi tensor product tools solving logical matrix, as in equation (11).

\section{Conclusion}

Pan Boolean algebra logic matrix design method of PID controller is proposed in this paper, the extended to general Pan Boolean algebra control, with the increase of the number of variables and the logic vector space growth, pan logic truth table expanded, manual calculation is very difficult. The solution is to solve the universal logic array design moment iterative algorithm, the establishment of universal logic really the value of this process and solving logic matrix completion by computer programming, this algorithm is very simple, because the process is two valued logic calculation, the computer is good at is two valued logic calculation.

\section{Acknowledgement}

In this paper, the research was sponsored by Shanghai Second Polytechnic University key subject construction project (No.XXKPY1308).

\section{References}

[1] Nanguan Zhang, Xiao Xian, Zhu Wujia, Pan Boolean algebra axiom system [J]. Journal of Air Force Institute of meteorology, 1985 (1): 338 353

[2] Zhang Nanguan, new control principle of [B], National Defence Industry Press, 2003.

[3]Jin Chen, Guanlin Chen, Zhenhua Wang, haihui Song, Jin Zhou, PID Pan Boolean Algebra Controller Parameters Space Analysis[C], Proceedings of the 30th Chinese Control Conference: 3840-3844,July 22-24, 2011, Yantai, China.

[4] Cheng DaiZhan, Qi Hongsheng, Zhao Yin, Boolean network analysis and control method of the semi tensor product of matrices [J],Journal of automation, 2011, Vol. 37 (5): 529-539.

[5]Chen, J., Zhou, J., Hong, X., Chen, G., Wang, Z.: PID Pan-Boolean algebra control. Journal of Central South University(Science and Technology)2009, 40(Sppl.1): 154-157 